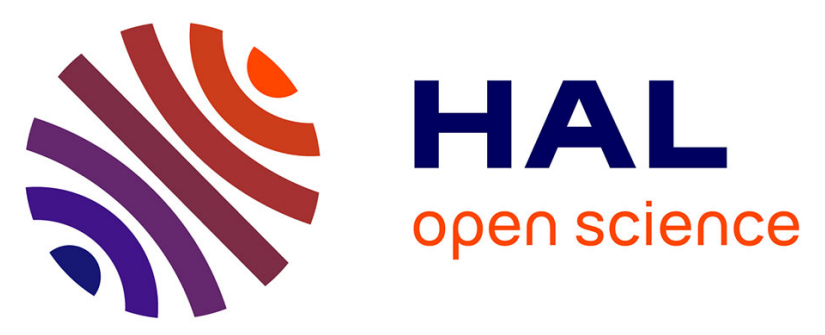

\title{
Reconstruction de la clavicule par la technique de la membrane induite chez l'enfant : à propos de 4 cas
}

Benjamin Haddad, Samuel Zribi, Elodie Haraux, François Deroussen, Richard Gouron, Céline Klein

\section{- To cite this version:}

Benjamin Haddad, Samuel Zribi, Elodie Haraux, François Deroussen, Richard Gouron, et al.. Reconstruction de la clavicule par la technique de la membrane induite chez l'enfant: à propos de 4 cas. Revue de Chirurgie Orthopédique et Traumatologique, 2019, 105, pp.493 - 498. 10.1016/j.rcot.2019.04.004 . hal-03486562

\section{HAL Id: hal-03486562 https://hal.science/hal-03486562}

Submitted on 20 Dec 2021

HAL is a multi-disciplinary open access archive for the deposit and dissemination of scientific research documents, whether they are published or not. The documents may come from teaching and research institutions in France or abroad, or from public or private research centers.
L'archive ouverte pluridisciplinaire HAL, est destinée au dépôt et à la diffusion de documents scientifiques de niveau recherche, publiés ou non, émanant des établissements d'enseignement et de recherche français ou étrangers, des laboratoires publics ou privés.

\section{(ㄷ)(1) $\$$}

Distributed under a Creative Commons Attribution - NonCommercial| 4.0 International 


\section{Mémoire original}

Reconstruction de la clavicule par la technique de la membrane induite chez l'enfant: à propos de 4 cas

Induced Membrane Technique for Clavicle Reconstruction in Paediatric Patients: Report of Four Cases

Benjamin Haddad $^{\mathrm{a}}$, Samuel Zribi ${ }^{\mathrm{a}}$, Elodie Haraux, ${ }^{\mathrm{a}}$, François Deroussen, ${ }^{\mathrm{a}}$, Richard Gouron, ${ }^{\text {a }}$, Céline Klein, ${ }^{\text {a }}$

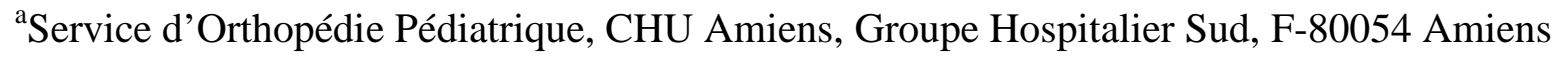
Cedex 1, FRANCE

Corresponding author: Dr Céline KLEIN, Service d'Orthopédie Pédiatrique, CHU Amiens, Groupe Hospitalier Sud, F-80054 AMIENS Cedex 1, France

Tel.: +33-322-087-570

Fax: +33-322-089-741

Email: celinekleinfr@yahoo.fr

Ne pas utiliser, pour citation, la référence française de cet article, mais celle de l'article original paru dans Orthopaedics \&Traumatology: Surgery \& Research, en utilisant le DOI ci-dessus.

\section{Résumé}

Introduction: Les reconstructions de la clavicule chez l'enfant sont rares et souvent complexes avec des résultats aléatoires. La technique de la membrane induite (MI) est une technique de reconstruction osseuse innovante en deux temps associant une interposition de ciment dans la zone réséquée, la formation d'une MI et une greffe cortico-spongieuse. 
Hypothèse: la reconstruction de la clavicule par la technique de la MI offre de bons résultats chez l'enfant.

Patients et méthodes: Quatre enfants ont été opérés d'une reconstruction de la clavicule par MI à l'âge moyen de 9,7 ans (7,4 - 12,3 ans). Il s'agissait d'une pseudarthrose congénitale de la clavicule $(n=3)$ et d'un kyste osseux anévrysmal. Au recul, la douleur et les mobilités de l'épaule ont été évaluées. Radiologiquement, la consolidation a été évaluée avec le score radiologique totale (SRT) allant de 0-10. Les complications et le nombre d'intervention par patient ont été notés.

Résultats: le recul moyen était de 3,9 ans (1-8,4 ans). Aucun patient ne présentait de douleur, ni de limitation articulaire. A 6 mois, toutes les clavicules étaient reconstruites et avaient un SRT à 10. Le nombre d'intervention était de 3,75/patients (3-5). On a noté deux reprises pour migration distale de broche et pour une collection sous cutané sur la broche.

Discussion: La membrane induite est une technique efficace pour reconstruire la clavicule avec peu de complications. Elle représente une alternative thérapeutique aux autres traitements.

Niveau de preuve : IV, série rétrospective

Mots clés: clavicule, enfant, membrane induite

\section{Introduction}

La reconstruction de la clavicule, quel qu'en soit l'étiologie, est un défi thérapeutique en terme de consolidation osseuse et de rétablissement de la longueur [1-7]. De nombreuses techniques (auto-greffe cortico-spongieuse, transfert de fibula...) ont été décrites avec des résultats très variables et aucune technique ne semble complètement fiable [1-7]. La 
technique de reconstruction par l'induction d'une membrane induite (MI) est une technique innovante réalisée en deux temps. Le premier temps consiste en l'interposition de ciment, poly methylmethacrylate (PMMA), induisant la formation d'une membrane autour du PMMA puis d'une greffe d'os cortico-spongieux à 6 semaines post-opératoire. Elle a été initialement utilisée pour les grandes pertes de substances osseuses diaphysaires chez l'adulte puis chez l'enfant [8-11] pour des reconstructions dans le cadre de résections tumorales, de pseudarthroses septiques ou congénitales. Elle semble offrir une bonne alternative thérapeutique avec d'excellents résultats en terme de consolidation et en particulier chez l'enfant, raisons pour lesquelles nous l'avons choisi pour la reconstruction de la clavicule [9]. Notre hypothèse de travail était que la technique de la MI assure une reconstruction efficace et durable pour les pertes de substances de clavicule chez l'enfant.

L'objectif primaire de notre étude était d'analyser les résultats cliniques et radiographiques à moyen et longs termes des patients traités par la technique de membrane induite de clavicule et de montrer les avantages et inconvénients de cette technique. Les objectifs secondaires étaient d'évaluer l'ostéosynthèse, les résultats en fonction de l'âge et de la lésion sous jacente.

\section{Matériel et méthodes}

\subsection{Patients}

Nous avons revu rétrospectivement toutes les données cliniques et radiologiques des patients pris en charge pour une reconstruction de la clavicule par la technique de MI entre mars 2010 et janvier 2018 dans le même centre. La revue des dossiers a été approuvée par la Commission Nationale de l'Information et des Libertés. Tous les patients et leurs parents ont été informés et ont donné leur consentement éclairé.

\subsection{Technique opératoire}


La technique opératoire était identique pour les quatre procédures. Les quatre patients ont été opérés par un chirurgien sénior. La chirurgie s'effectuait en deux temps. La première étape consistait en une résection de la zone de pseudarthrose et du kyste osseux qui allait de 2,9 cm à $6 \mathrm{~cm}$ par une voie longitudinale dans l'axe de la clavicule. Une broche de Kirschner centromédullaire était insérée en va et vient sous contrôle scopique puis une entretoise en polymethylmetacrylate (PMMA) Cerafixgenta (Ceraver Osteal, Roissy, France) était confectionnée à l'aide d'une seringue coupée en deux et positionnée entre les deux extrémités de clavicule en prenant soin de bien manchonner de quelques millimètre les deux extrémités. La seringue était ensuite facilement retirée après polymérisation du PMMA.

L'écart inter-fragmentaire claviculaire était maintenu à son maximum grâce au PMMA. Une immobilisation par coude-au-corps type Dujarrier était conservée pour une durée de six semaines. L'enfant était revu pour un contrôle radio-clinique à trois semaines post-opératoire. Le deuxième temps chirurgical se déroulait six semaines après le premier temps (Figure 1). Dans un premier temps, on prélevait les greffons cortico-spongieux pris aux dépens d'une crête iliaque postérieure. La voie d'abord de la clavicule était reprise et la membrane induite était incisée selon un axe longitudinal. Le ciment était enlevé par morcellement puis la cavité était comblée par l'autogreffe cortico-spongieuse éventuellement associée à une allogreffe par substitut osseux déminéralisé en copeaux pour le KOA. La MI était soigneusement refermée. La broche était ensuite retirée à distance une fois la consolidation osseuse acquise confirmée radiologiquement. Une antibioprophylaxie per-opératoire était instaurée pendant le premier et le second temps.

\subsection{Méthode}

Les données démographiques des patients ont été analysées. L'étiologie était répertoriée ((pseudarthrose congénitale de clavicule (PCC), kyste osseux anévrysmal (KOA)...). Les 
données cliniques des patients étaient notées lors de leur dernière consultation de contrôle et comprenaient la douleur évaluée subjectivement par l'échelle visuelle analogique (EVA), l'état de la cicatrice ainsi que les mobilités articulaires de l'épaule. Le nombre d'intervention et les complications étaient ensuite notés. Le résultat esthétique après chirurgie a été évalué verbalement par les patients et qualifié de minimum, modéré ou marqué [12].

L'analyse radiologique de la clavicule était réalisée en pré-opératoire avec la mesure de la distance inter-fragmentaire pour les PCC et la taille du KOA. Le suivi radiographique comportait une radiographie en post-opératoire immédiate, à 6 semaines, 3 mois, 6 mois et au dernier recul (Figure 2, Figure 3, Figure 4, Figure 5). Il a été évalué le délai de la consolidation, la recherche de fracture et de migration du matériel.

La consolidation osseuse était évaluée sur une échelle standardisée en dix points comprenant les moyennes des jonctions proximales et distales [13]. Nous avons utilisé le score radiographique total (SRT) qui correspond à la somme des moyennes des points pour la formation osseuse allant de 0 à 4 et pour la consolidation osseuse en proximale et distale allant de 0 à 3 pour chaque extrémité. La consolidation était acquise lorsque le SRT était compris entre 8 et 10 .

\section{Résultats}

Quatre patients ont pu être inclus. Tous les patients étaient de sexe féminin. Trois patients présentaient une PCC symptomatique et un patient présentait un KOA. La PCC était localisée au tiers moyen de la clavicule et à droite dans tous les cas. Elle était symptomatique à type de douleur à la mobilisation de l'épaule sans limitation des amplitudes articulaires et était jugée inesthétique par les trois patients. Le KOA de la clavicule était situé au tiers distal, côté gauche et le diagnostic était confirmé par une biopsie chirurgicale. 
L'âge moyen au moment de la chirurgie était de 9,7 ans (7,4 - 12,3 ans). Le recul moyen était de 3,9 ans (1-8,4 ans). Les résultats ont été rapportés dans le tableau 1.

Aucun patient ne présentait de douleur. Les amplitudes articulaires étaient complètes pour les quatre patients et comparables au côté controlatéral. Les cicatrices étaient non douloureuses, et une était hypertrophique au dernier recul. Les trois patients avec une PCC ont considéré le résultat esthétique comme marqué au dernier recul et le patient avec le KOA l'a considéré comme modéré. Concernant le site de prélèvement de la greffe cortico-spongieuse au niveau de la crête iliaque postérieure, aucun patient ne rapportait de douleur, ni de gêne esthétique liée aux cicatrices. Le nombre d'intervention moyen par patient était de 3,75 (3-5).

Trois patients $\left(\mathrm{n}^{\circ} 2, \mathrm{n}^{\circ} 3\right.$ et $\left.\mathrm{n}^{\circ} 4\right)$ présentaient des complications. Deux patients ont été réopérés deux semaines après le second temps d'une recoupe de broche suite à une extériorisation distale. Un patient a présenté un abcès sous cutané en regard de l'orifice de sortie de la broche un mois après le deuxième temps. Deux fractures suite à des traumatismes de faible intensité ont été constatées chez le patient $\mathrm{n}^{\circ} 2$, une première au niveau de la greffe (à 4 mois du second temps) puis à la jonction distale greffe-quart latéral (à 7 mois du second temps) qui ont toutes deux été traitées par une immobilisation par coude-au-corps et ont consolidé en un mois. Une dernière intervention a été pratiquée chez ce même patient 2,5 ans après le second temps pour réséquer un spicule osseux gênant. Il n'a pas été observé de récidive du KOA.

A 3 mois du deuxième temps, le SRT était compris entre 8 et 10 dans les 3 cas et à 7 pour un cas. Le SRT était à 10 à 6 mois de l'intervention dans tous les cas.

\section{Discussion}

Notre travail confirme que la MI est une technique de reconstruction de la clavicule qui permet une reconstruction satisfaisante et durable avec de bons résultats cliniques et radiologiques chez l'enfant. A ce jour, il n'existe pas de consensus pour la reconstruction de la 
clavicule chez l'enfant. Seuls deux cas de reconstruction de la clavicule en deux temps par la technique de la MI ont été publiés dont un fait partie de l'actuelle série [14,15].

Notre étude présentait plusieurs limites dont le caractère rétrospectif et la faiblesse de notre échantillon n'autorisant pas d'analyse statistique ainsi que l'absence de groupe contrôle. De plus, nos patients sont jeunes à la revue et un recul plus long serait nécessaire.

Nous avons choisi cette technique de reconstruction par MI car elle a l'avantage d'offrir de bons résultats chez l'enfant avec peu de morbidité [11,16,17] bien que les propriétés d'angiogenèse, d'ostéo-induction et d'ostéogénèse de la membrane induite demeurent à ce jour non encore toutes élucidées $[8,10]$. Le principal inconvénient lié à la technique de la MI est la nécessité d'un deuxième temps chirurgical. Les autres inconvénients et complications notés sont liés à la reconstruction de la clavicule et des moyens d'ostéosynthèse de cette région.

Les autres techniques de reconstruction de la clavicule chez l'enfant ont essentiellement porté sur les PCC [1-5,18]. Différentes techniques de reconstruction sont envisageables mais l'ensemble des études s'accorde sur l'importance de l'auto greffe cortico-spongieuse. Di Gennaro [2] a reporté 27 cas de PCC avec un âge moyen de 8,1 ans et sur les 19 patients opérés par résection-greffe en un temps, 15 ont eu une auto greffe cortico-spongieuses, 5 n'ont pas consolidé dans un premier temps dont 3 avaient eu une allogreffe en copeaux osseux déminéralisés. Il a aussi rapporté 4 allogreffes de fibula avec de mauvais résultats dont 2 absences de consolidation et une résorption de la greffe. Cadhilac et al. [1] ont étudié 17 cas de PCC avec une moyenne d'âge de 6 ans et 4 mois, traités par plaque dont 9 ont été greffés avec une crête iliaque et 8 n'ont pas été greffés. Tous les patients greffés ont consolidés alors que seulement 5 des 8 non greffés ont consolidé ce qui était en faveur de la nécessité d'une auto-greffe quel que soit le moyen de fixation. Eliott and Richards [18] ont montré 2 échecs de reconstruction claviculaire pour PCC après xénogreffe bovine avec la survenue d'une lyse complète de la greffe et ont donc déconseillé l'utilisation de ce produit. 
Néanmoins, Grogan et al. [5] a reporté sur une série de 8 PCC traitées par excision de la zone de pseudarthrose et cerclage au fil sans greffe ni fixation rigide par plaque ou broche et a retrouvé une consolidation dans $100 \%$ des cas. Il faut noter que 6 patients parmi les 8 avaient un âge inférieur à 2,5 ans ce qui peut expliquer l'absence d'utilisation d'une greffe osseuse mais nous nous questionnons sur la récupération de la longueur de la clavicule. Un transfert de fibula vascularisé a été décrit dans le cadre d'une reprise chirurgicale après lyse massive du greffon avec une ostéointégration parfaite à 7 mois [7]. Néanmoins, la rançon cicatricielle et peut être fonctionnelle pour le site donneur est important. Bien que les suites semblent identiques, nous utiliserions uniquement ce procédé pour les reprises chirurgicales en cas d'échec d'une MI.

A ce jour, aucun mode de fixation ne semble idéal. En effet dans notre série, nous avons utilisé des broches et observé des extériorisations de broches nécessitant des recoupes. Chandran a comparé deux méthodes de fixations (broche vs plaque) et a retrouvé plus de pseudarthroses dans le groupe broche que dans le groupe plaque (2 vs 0) [4]. En revanche, Persiani, a comparé 8 reconstructions de clavicules par broches à 9 par plaque et a obtenu des résultats contradictoires à ceux de Chandran [3,4]. Dans une revue de la littérature, Huntley a recommandé chez l'enfant de moins de 4 ans de réaliser une greffe cortico-spongieuse sans ostéosynthèse et après 4 ans de préférer les plaques aux broches [6]. A la vue de nos résultats et de ceux de la littérature, il semblerait que l'utilisation de plaque permette de diminuer les complications mais expose au risque de bris de matériel, de gêne sous cutanée et impose une nouvelle intervention pour retirer le matériel.

Dans notre série, même si notre effectif reste modeste, nous n'avons pas trouvé de différence en terme de consolidation et de complications selon l'âge. Il faut noter que l'âge idéal pour effectuer une reconstruction de clavicule n'est pas consensuel. La plupart des auteurs 
recommandent une chirurgie précoce chez des patients avant 5 ans, voir le plus tôt possible $[3,5,19]$.

Néanmoins, l'étiologie de la lésion semble influencer les résultats. Le KOA est une lésion rare et sa localisation claviculaire l'est encore plus et se situe essentiellement au niveau du quart latéral [19]. Kaiser et al. ont observé un important risque de récurrence en cas de curetage avec ou sans greffe. Cependant, Bakkaly et al. ont retrouvé un bon résultat avec un remodelage osseux et l'absence de récidive à propos d'un cas de KOA situé au quart latéral de clavicule chez un enfant de 8 ans traité par résection sans reconstruction [20]. Pour notre cas de KOA, nous avions choisi la technique de la MI qui offrait une reconstruction plus anatomique avec un bon résultat en terme de consolidation mais au prix de complications fracturaires. Plus que l'étiologie, il semblerait que la localisation latérale du KOA explique le nombre important de complications.

\section{Conclusion}

La reconstruction de la clavicule par la technique de la membrane induite est une alternative thérapeutique envisageable, offrant de bons résultats en terme de consolidation avec peu de complications au prix d'une reconstruction en deux étapes.

\section{Déclaration de liens d’intérêts}

Les auteurs déclarent ne pas avoir de liens d'intérêts.

Sources de financement : Aucune

\section{Contribution des auteurs :}

B Haddad and C Klein : rédaction du manuscrit

R Gouron and E Haraux : révision du manuscrit 
S Zribi and F Deroussen : collecte des données 


\section{Références}

[1] Cadilhac C, Fenoll B, Peretti A, Padovani JP, Pouliquen JC, Rigault P. Congenital pseudarthrosis of the clavicle: 25 childhood cases. Rev Chir Orthop 2000;86:575-80.

[2] Di Gennaro GL, Cravino M, Martinelli A, Berardi E, Rao A, Stilli S, et al. Congenital pseudarthrosis of the clavicle: a report on 27 cases. J Shoulder Elbow Surg 2017;26:65-70.

[3] Persiani P, Molayem I, Villani C, Cadilhac C, Glorion C. Surgical treatment of congenital pseudarthrosis of the clavicle: a report on 17 cases. Acta Orthop Belg 2008;74:161-6.

[4] Chandran P, George H, James LA. Congenital clavicular pseudarthrosis: comparison of two treatment methods. J Child Orthop 2011;5:1-4.

[5] Grogan DP, Love SM, Guidera KJ, Ogden JA. Operative treatment of congenital pseudarthrosis of the clavicle. J Pediatr Orthop 1991;11:176-80.

[6] Huntley JS. Evidence-Based Treatment of Congenital Clavicular Pseudarthrosis. In: Alshryda S, Huntley JS, Banaszkiewicz PA, editors. Paediatric Orthopaedics, Cham: Springer International Publishing; 2017, p. 279-85.

[7] Glotzbecker MP, Shin EK, Chen NC, Labow BI, Waters PM. Salvage reconstruction of congenital pseudarthrosis of the clavicle with vascularized fibular graft after failed operative treatment: a case report. J Pediatr Orthop 2009;29:411-5.

[8] Masquelet AC, Begue T. The concept of induced membrane for reconstruction of long bone defects. Orthop Clin North Am 2010;41:27-37.

[9] Gouron R, Deroussen F, Plancq M-C, Collet L-M. Bone defect reconstruction in children using the induced membrane technique: a series of 14 cases. Orthop Traumatol Surg Res 2013;99:837-43.

[10] Chotel F, Nguiabanda L, Braillon P, Kohler R, Bérard J, Abelin-Genevois K. Induced membrane technique for reconstruction after bone tumor resection in children: A preliminary 
study. Orthopaedics \& Traumatology: Surgery \& Research 2012;98:301-8.

[11] Pannier S, Pejin Z, Dana C, Masquelet AC, Glorion C. Induced membrane technique for the treatment of congenital pseudarthrosis of the tibia: preliminary results of five cases. $\mathbf{J}$ Child Orthop 2013;7:477-85.

[12] Swanson AB, Hagert CG, Swanson GD. Evaluation of impairment of hand function. J Hand Surg Am 1983;8:709-22.

[13] Johnson KD, Frierson KE, Keller TS, Cook C, Scheinberg R, Zerwekh J, et al. Porous ceramics as bone graft substitutes in long bone defects: a biomechanical, histological, and radiographic analysis. J Orthop Res 1996;14:351-69.

[14] Gouron R, Deroussen F, Juvet-Segarra M, Plancq M-C, Collet L-M. Reconstruction of Congenital Pseudarthrosis of the Clavicle with Use of the Masquelet Technique: A Case Report. JBJS Case Connect 2012;2:77.

[15] Abdellaoui H, Atarraf K, Chater L, Afifi MA. Congenital pseudarthrosis of the clavicle treated by Masquelet technique. BMJ Case Rep 2017;8.

[16] Biau DJ, Pannier S, Masquelet AC, Glorion C. Case report: reconstruction of a 16-cm diaphyseal defect after Ewing's resection in a child. Clin Orthop Relat Res 2009;467:572-7. [17] Gouron R, Deroussen F, Juvet M, Ursu C, Plancq MC, Collet LM. Early resection of congenital pseudarthrosis of the tibia and successful reconstruction using the Masquelet technique. J Bone Joint Surg Br 2011;93:552-4.

[18] Elliot RR, Richards RH. Failed operative treatment in two cases of pseudarthrosis of the clavicle using internal fixation and bovine cancellous xenograft (Tutobone). J Pediatr Orthop B 2011;20:349-53.

[19] Gibson DA, Carroll N. Congenital pseudarthrosis of the clavicle. J Bone Joint Surg Br 1970;52:629-43.

[20] Bakkaly AE, Hanine MD, Amrani A, Dendane A, El Alami SZF, Madhi TE. Kyste 
anévrismal osseux de la clavicule: à propos d'un cas. Pan Afr Med J 2017;27. 
Tableau 1: Données des patients incluant la démographie, le type et la localisation de la pathologie, le côté, l'écart inter fragmentaire initial et le déficit osseux à combler, la durée du recul, le nombre et le type de complications et le résultat final

\begin{tabular}{|c|c|c|c|c|c|c|c|c|c|c|c|}
\hline Patient & Sexe & $\begin{array}{c}\text { Âge lors de } \\
\text { la chirurgie } \\
\text { (mois) }\end{array}$ & Côté & Localisation & Pathologie & $\begin{array}{c}\text { Espace inter- } \\
\text { fragmentaire avant } \\
\text { résection }\end{array}$ & $\begin{array}{c}\mathrm{Nb} \\
\text { inter- } \\
\text { vention }\end{array}$ & $\begin{array}{l}\text { Taille } \\
\text { zone } \\
\text { réséquée }\end{array}$ & $\begin{array}{l}\text { Recul } \\
\text { en année }\end{array}$ & Complications & Résultat final \\
\hline 1 & $\mathrm{~F}$ & 7,4 & $\mathrm{D}$ & $1 / 3$ moyen & PCC & 6 & 3 & 35 & 8,4 & 0 & $\mathrm{C}$ \\
\hline 2 & F & 12,3 & G & $1 / 3$ lat & KOA & NA & 5 & 60 & 5,1 & $\begin{array}{l}\text { Recoupe de broche } \\
\quad 2 \text { fractures } \\
\text { Exérèse de spicule }\end{array}$ & $\mathrm{C}$ \\
\hline 3 & $\mathrm{~F}$ & 9,5 & D & $1 / 3$ moyen & PCC & 7 & 4 & 29 & 1,2 & Recoupe de broche & $\mathrm{C}$ \\
\hline 4 & $\mathrm{~F}$ & 9,5 & D & $1 / 3$ moyen & PCC & 25 & 3 & 33 & 1 & Abcès sous-cutané & $\mathrm{C}$ \\
\hline
\end{tabular}

Légendes: PCC pseudarthrose congénitale clavicule, KOA kyste osseux anevrysmal, LAT latéral, NA non applicable, C consolidation 


\section{Légendes}

Figure 1: Patient 3 - Photographies per-opératoires du deuxième temps chirurgical de la membrane induite. Cicatrice pré-opératoire (A), membrane induite avant incision (B), membrane induite incisée horizontalement (flèche large) et PMMA (flèche fine) (C), ablation du PMMA et visualisation de la broche préalablement insérée lors du premier temps (D).

Figure 2: Patient 1 - Reconstruction de clavicule dans le cadre d'une pseudarthrose congénitale de clavicule droite. Radiographie pré-opératoire (A). Radiographie postopératoire immédiat du premier temps (B), du deuxième temps (C), au dernier recul (D) montrant une bonne consolidation osseuse à 8,4 ans.

Figure 3 : Patient 2 - Reconstruction de clavicule dans le cadre d'un kyste osseux anévrysmal du quart latéral de la clavicule gauche. Radiographie pré-opératoire (A), radiographie postopératoire immédiat du premier temps $(\mathrm{B})$, du deuxième temps $(\mathrm{C})$, au dernier recul montrant un bon remodelage osseux à 5,1 ans (D).

Figure 4: Patient 3 - Reconstruction de clavicule dans le cadre d'une pseudarthrose congénitale de clavicule droite. Radiographie pré-opératoire (A), radiographie post-opératoire immédiat du premier temps (B), du deuxième temps (C), au dernier recul à 1,2 ans (D).

Figure 5: Patient 4 - Reconstruction de clavicule dans le cadre d'une pseudarthrose congénitale de clavicule droite. Radiographie pré-opératoire (A), radiographie post-opératoire immédiat du premier temps $(\mathrm{B})$, du deuxième temps $(\mathrm{C})$, au dernier recul à un an $(\mathrm{D})$. 

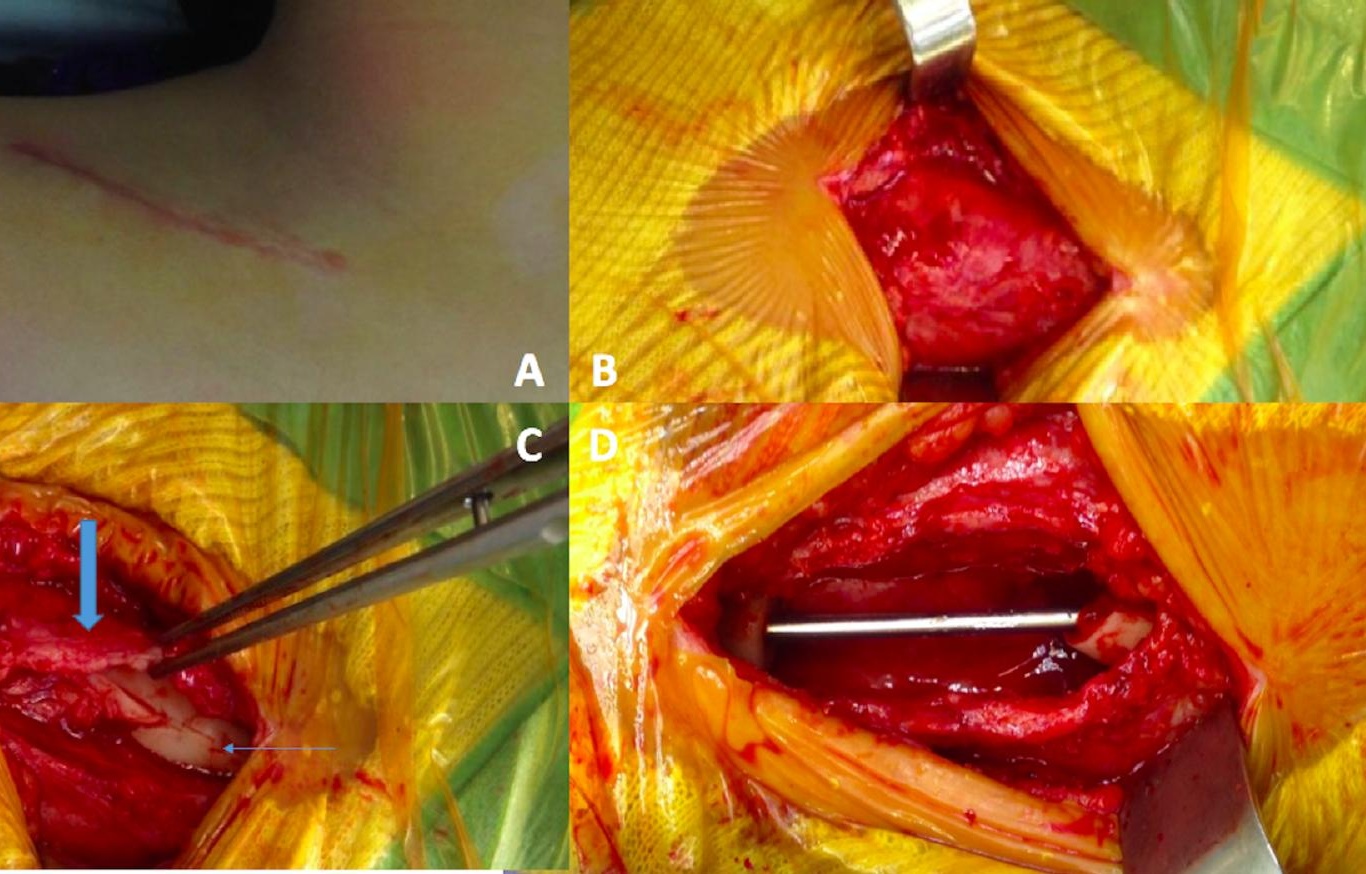


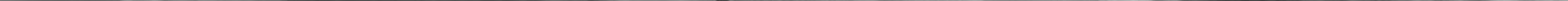


de

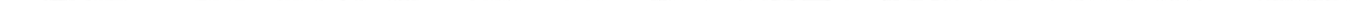

te

पit

ain

A B

C D 
D

(D)

C D

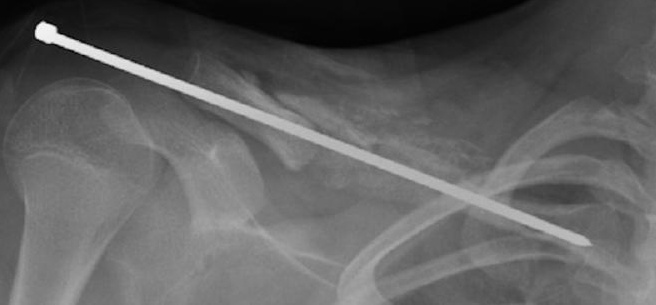




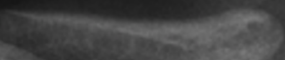

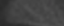

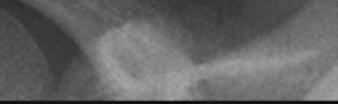

2
A

C D
B 